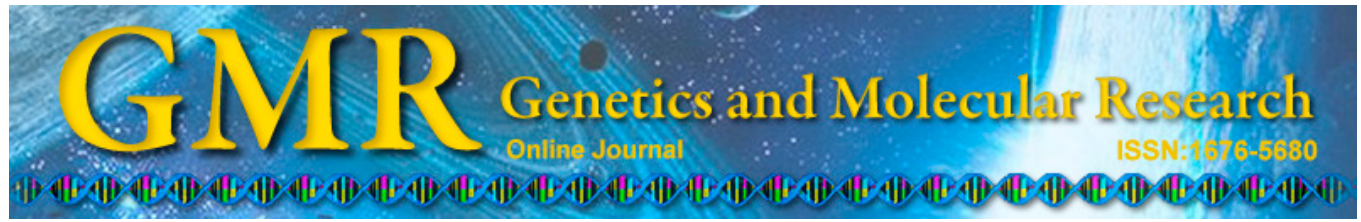

\title{
Specialized features of the outer hair cell shapes in the cochlear fovea of bats
}

\author{
S.Q. Zhang' ${ }^{1}$ S.L. Li ${ }^{2}$, H.L. Zhü ${ }^{2}$ and L.Y. Yan ${ }^{1}$ \\ ${ }^{1}$ Department of Otolaryngology, The First Affiliated Hospital, \\ Medicine School of Xi' an Jiaotong University, Xi' an, China \\ ${ }^{2}$ Center for Research Laboratory, The Second Affiliated Hospital, \\ Medicine School of Xi' an Jiaotong University, Xi'an, China \\ Corresponding author: S.Q. Zhang \\ E-mail: shaoqiangzhang_cn@163.com
}

Genet. Mol. Res. 14 (3): 9530-9542 (2015)

Received November 25, 2014

Accepted April 6, 2015

Published August 14, 2015

DOI http://dx.doi.org/10.4238/2015.August.14.16

\begin{abstract}
In this study, we examined the specialized features of the outer hair cells (OHCs) and the stereocilium bundles of the bat cochlear fovea. Bat cochlea hair cells were observed by scanning and transmission electron microscopy, and the auditory brainstem response thresholds were assessed. The stereocilia bundles of the OHCs were extremely short. The OHC bodies were flask-shaped and cambiform or ball-shape in the cochlear fovea. Digitations in the Deiters cells had exaggerated lengths, and cup formation of the Deiters cell, housed at the bottom of the OHC in the base of the cell, showed a specialized shape. Our results provide the first evidence that different shapes of the OHCs in the cochlea fovea are related to the high-frequency function of auditory response. Echolocating bats have cochlear morphologies that differ from those of non-echolocating animals. Bat cochlear foveae are specialized for analyzing the Doppler-shifted echoes of the first-harmonics of the $\mathrm{CF}_{2}$ component; these are overrepresented in the frequency range around the dominant harmonic of the echolocation calls of bats. However, the $\mathrm{OHCs}$ of the bat cochlear fovea have not been fully characterized.
\end{abstract}

Key words: Bat; Cochlea fovea; Echolocation; Outer hair cells; Stereocilia 


\section{INTRODUCTION}

Bats are the only flying mammals (Kirkegaard and Jørgensen, 2000), and because of their need for echolocation, most bats have a very specialized organ of Corti so that highfrequency sounds are audible (Macías et al., 2006). In the ultrasonic range, bat audiograms typically show the highest sensitivity within the spectral region of their species-specific echolocation calls (Bohn et al., 2006). These species are characterized by a resonance mechanism in the basal region of the cochlea, with extremely fine-tuned properties where important parts of the sonar signal are processed. This area, known as the acoustic fovea (Russell and Kössl, 1999), amplifies the tuning that arises from an overrepresentation of the narrow frequency band in the cochlear fovea. The cochlear fovea displays a widely expanded frequency map. The innervation density per octave is significantly high in this region, where $25 \%$ of the receptors and $21 \%$ of the spiral ganglion neurons of the cochlea represent $10 \%$ of a single octave (Bruns and Schmieszek, 1980). The horseshoe bats (Rhinolophus ferrumequinum) and leaf-nosed bats (Hipposideros spp) both possess auditory foveae and emit constant-frequency calls (CFs) (Li et al., 2008). The presence of the fovea region of the cochlea, as well as that of the specialized basal apical gradients in the morphology of the basilar (BM) and tectorial membranes (TM), which are essential components of the acoustic laser, are established. The fovea region of the mustached bat cochlea functions as an acoustic laser beginning at birth (Russell and Kössl, 1999). Specialized features in the cochlear inner hair cells (IHCs) and outer hair cells (OHCs) are required for echolocation. In contrast, the basal OHCs from the cochlea of the horseshoe bat, as observed by electron microscopy, are cylindrical (Vater et al., 1992). The cochlea of the Mexican free-tailed bat (Tadarida brasiliensis) has cylindrical OHCs (Vater and Siefer 1995). Mammalian OHCs are slender cylindrical structures with fairly uniform diameters (8-10 m) (Dallos et al., 1991). In mustached bats, the cochlear microphonic potential (CM) mainly generated by OHCs is sharply tuned to 61 $\mathrm{kHz}$, although the increase in the duration of $\mathrm{CM}$ after stimulation suggests sharpening of the frequency tuning (Suga et al., 2000). However, little is known about the specialized features of the cochlear hair cells in these mammals, particularly in the bat cochlea. A previous study indicated that the shape of atypical OHCs conforms to the typical mammalian shape, whereas basal OHCs are much shorter and ball-shaped. These variations in shape may have resulted from an increase in turgidity following exposure to a hypoosmotic extracellular solution (Reuter et al., 1994).

In this study, we examined bat $\mathrm{OHCs}$ on an ultrastructural level to provide a database for comparison between OHCs in cochleae designed to hear in the auditory fovea region and those in the cochleae of non-echolocating mammals.

\section{MATERIAL AND METHODS}

\section{Ethics statement}

All animals were maintained in accordance with protocols approved by the Ethical Committee of the First Affiliated Hospital, School of Medicine, Xi'an Jiaotong University and approved by the Zhashui Forestry Bureau (No. 2009017). 


\section{Subjects}

A total of 12 bats were examined. Twelve left and twelve right cochleae were used for scanning electron microscopy (SEM) and transmission electron microscopy (TEM) examination, respectively. Adult bats from 4 species, including Hipposideros pratti Thomas, Rhinolophus rouxi sinicus Andersen, Miniopterus schreibersii chinensis Thomas, and Molossidae Tadarida, from a cave in Zhashui, China were examined (Table 1). The cave is located in Qinling Mountain of Shaanxi Province, China. The species were identified by the Institute of Zoology, Chinese Academy of Sciences, Beijing.

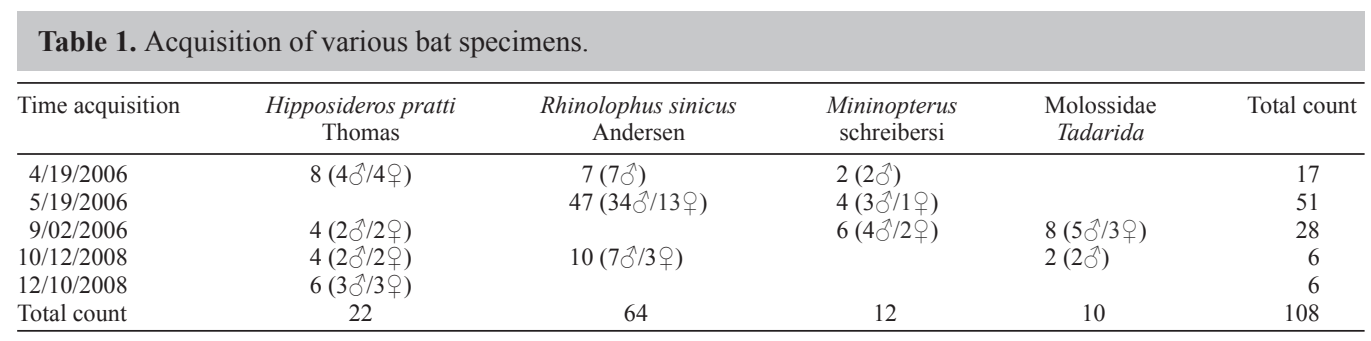

The animals were individually housed and given free access to water and insect food, which included crickets and locusts, during daily test sessions.

\section{Auditory brainstem response (ABR) methodologies}

Stimuli were synthesized using the TDT System 3 hardware and software (TuckerDavis Technologies, Alachua, FL, USA), as well as an electrostatic loudspeaker speaker (Tucker-Davis Technologies) with a relatively flat frequency response from 2-100 kHz. The speaker was mounted $20 \mathrm{~cm}$ in front of the bat's head in the free field inside a foam-padded and shielded acoustic chamber. A mouse was placed in the prone position facing a broadband electrostatic loudspeaker system (TDT ES1 with ED1 speaker driver). Miniature subdermal needle electrodes (Nicolet; Natus Medical Incorporated, Pleasanton, CA, USA) were inserted at the vertex (reference), over the bulla (active), and just above the hind nose (ground). Calibrated tone bursts ( $5 \mathrm{~ms}$ duration, $0.5 \mathrm{~ms}$ rise-fall time, phase alternating $90 \mathrm{~nm}$ ) were synthesized using the SigGenRP* software on a TDT RP2.1 real-time processor and presented at a rate of $49 / \mathrm{s}$ at frequencies of $2,4,8$, and up to $100 \mathrm{kHz}$ at $4 \mathrm{kHz}$ increments. The amplitude of the signal was automatically attenuated (TDT PA5) in 5-dB steps at each frequency. The amplified digital signal was sent to a Pentusa RX5-2 base station via optic fibers. The signal was filtered (3-300 Hz for middle latency response recording and 300-3000 Hz for ABR recording) and averaged using the TDT BioSig software. The acoustic stimuli were tone bursts $(5 \mathrm{~ms}$ duration with $2 \mathrm{~ms}$ rise-fall times) with decreasing intensity (5-dB decrements) from $100 \mathrm{~dB}$ sound pressure level (SPL) to $0 \mathrm{~dB}$ SPL over a frequency range of 1-100 $\mathrm{kHz}$.

The ABR results were collected using a Tucker-Davis Technologies BioSig System. $A B R$ were recorded with subcutaneous platinum needle electrodes placed at the vertex, right mastoid prominence, and tail. Multiple tone burst sequences were presented at various sound pressure levels over the attenuation range of $100-0 \mathrm{~dB}$ in $5-\mathrm{dB}$ decrements. The responses to 
the tone pips were amplified by $80 \mathrm{~dB}$, bandpass filtered $(0.3-3 \mathrm{kHz})$, and the sound stimuli were digitally generated, attenuated using programmable attenuators, and delivered via electrostatic speakers (RP-2, PA-5, ED-1, and EC-1; Tucker-Davis Technologies).

\section{SEM}

Under deep pentobarbital anesthesia $(60 \mathrm{mg} / 100 \mathrm{~g})$, the bats were decapitated, and their cochlea were quickly removed from the skull and immersed in fixative solution composed of $2.5 \%$ glutaraldehyde in $0.1 \mathrm{M}$ phosphate buffer. The right cochleae of the bats were observed by SEM, whereas the left cochleae were examined by TEM. The round and oval windows were immediately opened and a small hole was drilled into the apex $\left(\mathrm{CF}_{2}\right)$ and basal (hook) turns. The fixative solution was perfused through the cochlea via the oval or round window and the small hole. The specimens were post-fixed in the same solution for $3 \mathrm{~h}$ to several days and stored below $4^{\circ} \mathrm{C}$. After rinsing in buffer, the cochleae were micro-dissected using a surgical microscope. The bone, vestibular membrane, and TM were removed to expose the receptor surface of the cochlear fovea. The cochlea was then treated with $1 \% \mathrm{OsO}_{4}$ for 1 $\mathrm{h}$, rinsed, and then dehydrated in an increasing alcohol gradient (30-100\%). The BM was exposed, and the critical point was dried and coated with gold. All specimens were viewed using SAG (styrene-acrylon-glycidyl methacrylate):40X SEM. The cochlear foveae of the bats were also observed.

\section{TEM}

Cochleae from 13 bats were fixed by intracochlear perfusion with 3\% glutaraldehyde in cacodylate buffer. The specimens were decalcified in EDTA for 4 days. The cochleae were post-fixed with $4 \% \mathrm{OsO}_{4}$ and microdissected using a surgical microscope. The bone, vestibular membrane, and the TM were removed. The BM was removed, dehydrated, and embedded in Durcupun. Ultrathin sections were cut, stained with uranyl acetate and lead citrate, and observed under a JEM-1200EX TEM (Jeol, Ltd., Tokyo, Japan).

\section{RESULTS}

\section{SEM}

Bats were fixed on the recording station in the free field inside a foam-padded and shielded acoustic chamber. The bat cochlea showed the typical mammalian shape, which consisted of 3 turns, the $\mathrm{CF}_{2}$, middle (SI), and basal turn (hook). The BM of the bat cochlea was wider than that of non-echolocating mammals (Figure 1). In a camera lucida drawing of the whole mount of the cochlear hook turn, with afferent nerve fibers and the spiral ligament, 2 densely innervated audiograms (hook) were separated by a sparsely innervated zone (SI region). At the transition between the $\mathrm{SI}$ and $\mathrm{CF}_{2}$ regions, the spiral ligament was enlarged. In the SI zone, the IHCs were tuned to frequencies from $62-72 \mathrm{kHz}$.

The basal turn (hook) IHC possessed only 1-2 rows of stereocilia and only 6-8 stereocilia per row (Figure 2). The cuticular plates were small and widely separated with 14-20 stereocilia per row. The cuticular plates were elongated and closely spaced. The OHCs were extremely wide, W-shaped, with shortened stereocilium bundles, and wing-shaped cuticular 
plates in the $\mathrm{SI}$ to $\mathrm{CF}_{2}$ regions. The cuticular plates of the $\mathrm{OHC}$ of the middle and outermost rows were arranged parallel to each other.

Scanning electron micrographs of the bat $\mathrm{OHC}$ revealed shortened stereocilium
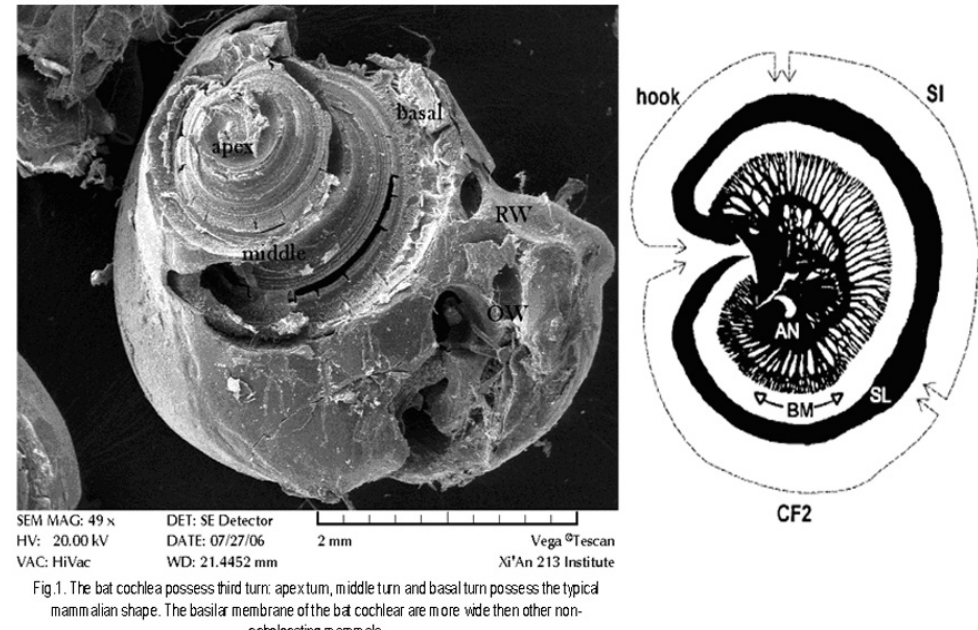

mammalian shape. The basila membrane of the bł cochlear are more wde then other nonectrolocating mammals.

Figure 1. Cochlea structure. The bat cochlea exhibited the typical mammalian shape, which consists of 3 turns: $\mathrm{CF}_{2}$, SI, and hook turns. The BM of the bat cochlea was wider than that of non-echolocating mammals.
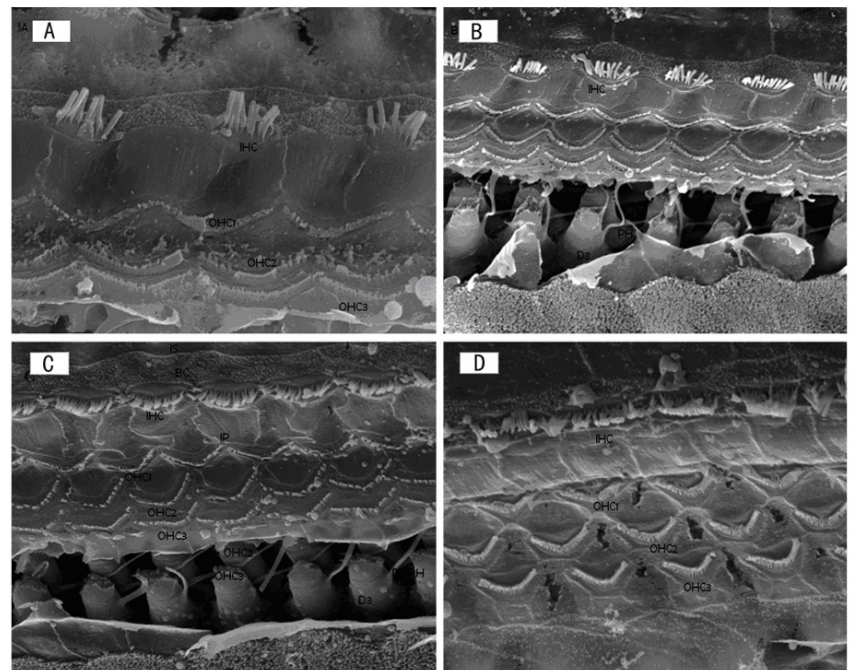

Figure 2. Scanning electron micrographs of IHC and OHC. Photomicrographs of the bat cochlear IHC and OHC. (A-D) Scanning electron micrographs of the organ of Corti from basal turn to apex turn. A.-B. Basal turn (hook) IHC possessed only 1-2 rows of stereocilia, with only 6-8 stereocilia per row. The cuticular plates were small and widely separated; C.-D. 14-20 stereocilia per row, and the cuticular plates were elongated and closely spaced. The $\mathrm{OHCs}$ were extremely wide and W-shaped, with shortened stereocilia bundles and wing-shaped cuticular plates in the $\mathrm{SI}$ to $\mathrm{CF}_{2}$ regions. A.-C. Cuticular plates of the $\mathrm{OHC}$ of the middle and outermost rows were arranged parallel to each other (B-D). 
bundles. The $\mathrm{OHC}$ in the apex turn $\left(\mathrm{CF}_{2}\right.$ regions) exhibited a cylindrical body, whereas the OHCs in middle turn (SI regions) were round and flask-shaped (Figure 3). Three rows of OHCs were housed at the bottom of the Deiters cell. The Deiters cell phalanges had exaggerated lengths. The $\mathrm{OHC}$ in the middle (SI regions) turn was flask-shaped with extremely short stereocilium bundles. Another $\mathrm{OHC}$ in the basal turn was rounded. The specialized cup shape of the Deiters cell body housed the bottom of the 3 rows of OHCs (Figures 4 and 5). SEM revealed that the $\mathrm{OHCs}$ of the $\mathrm{BALB} / \mathrm{c}$ mouse cochleae were cylindrical, with extremely short stereocilium bundles, whereas the cochlear $\mathrm{OHC}$ of guinea pigs had exaggerated lengths (Figure 6). The C57/BJ, B6/J, and NMF308 mouse cochlear OHC bodies were cylindrical and extremely long (Figure 7). (BALB/c, C57/BJ, B6/J and NMF308 mouse cochlear were based on our unpublished data).

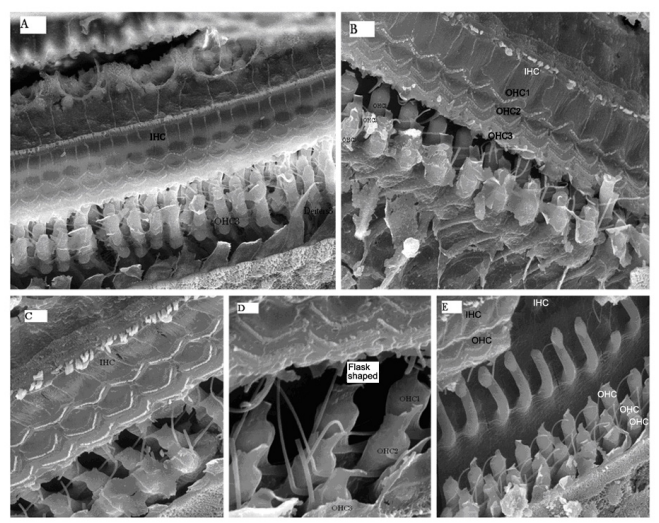

Figure 3. Scanning electron micrographs of stereocilium bundles. Scanning electron micrographs exhibited an $\mathrm{OHC}$ with shortened stereocilium bundles. A.-B. The apex turn $\left(\mathrm{CF}_{2}\right.$ regions $)$ of $\mathrm{OHC} 3$ exhibited a cylindrical body. C.-E. The SI regions of $\mathrm{OHC1}, \mathrm{OHC} 2$, and $\mathrm{OHC} 3$ exhibited round and flask shapes.

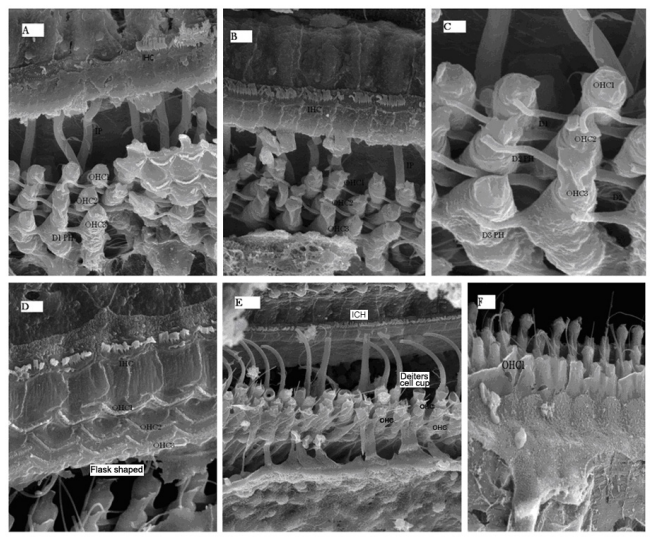

Figure 4. Scanning electron micrographs of the OHCs and their connection to the Deiters cells. A. Three rows of OHC were housed at the bottom of the Deiters cell. A.-B. Deiters cell phalanges had exaggerated lengths. C. The connection point between the 3 rows of OHCs and 3 rows of Deiters cells. D. Basal region (SI regions) of the OHCs were flask-shaped. E. Specialized cup shape of the Deiters cell body, which housed the bottom of the 3 rows OHC. F. OHC in the basal turn was round. 


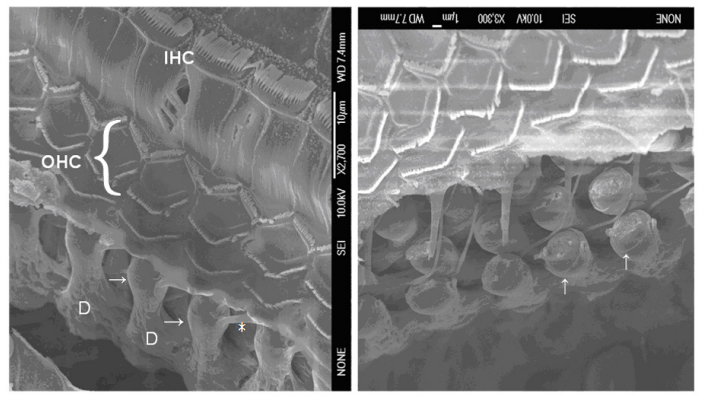

Figure 5. Scanning electron micrographs of the OHCs in the bat cochleae. The OHCs were ball-shaped and flaskshaped, with extremely short stereocilia bundles (arrows in SI regions). D = Dieters cell; IHC = inner hair cell.
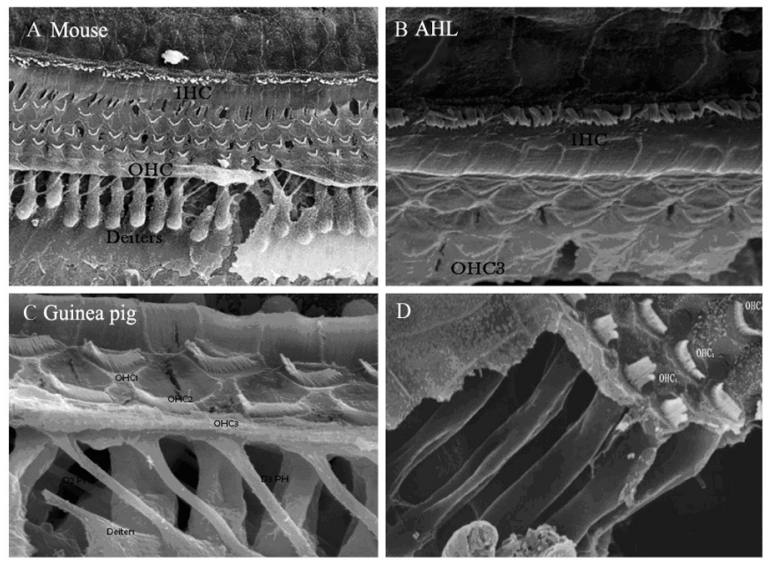

Figure 6. Scanning electron micrographs of the OHCs in the BALB/c mouse cochleae. The OHCs of the BALB/c mouse cochleae were cylindrical, with extremely short stereocilia bundles. The cochlear OHCs of the guinea pig had exaggerated lengths.

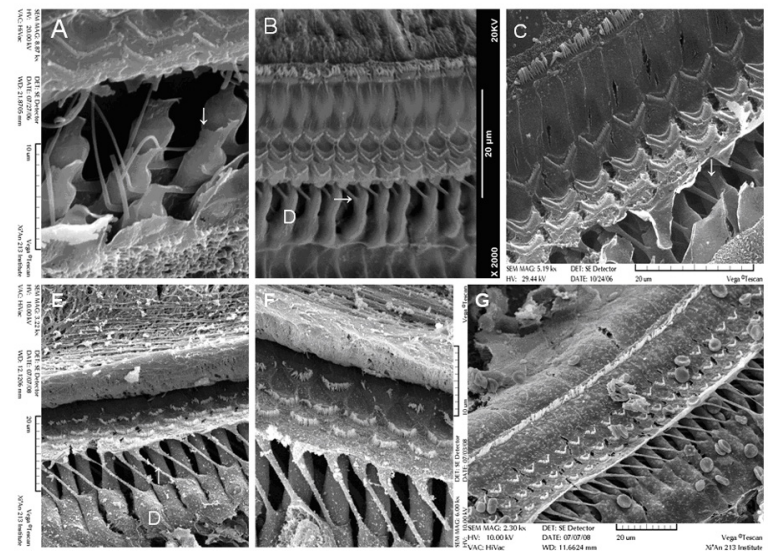

Figure 7. Scanning electron micrographs of the OHCs of bats. The OHCs of bats (A-C) were round and flaskshaped in the SI region; D. Dieters cell; C57/BJ (E), B6/J (F), and NMF308 mouse (G) cochlear OHC bodies were cylindrical and extremely long. 


\section{TEM}

The guinea pig cochlea OHC1s were slender and cylindrical. The Microtus mandarinus $\mathrm{OHCs}$ were also cylindrical, and the location of the nucleus was in its normal position near the basal pole (Figure 8).

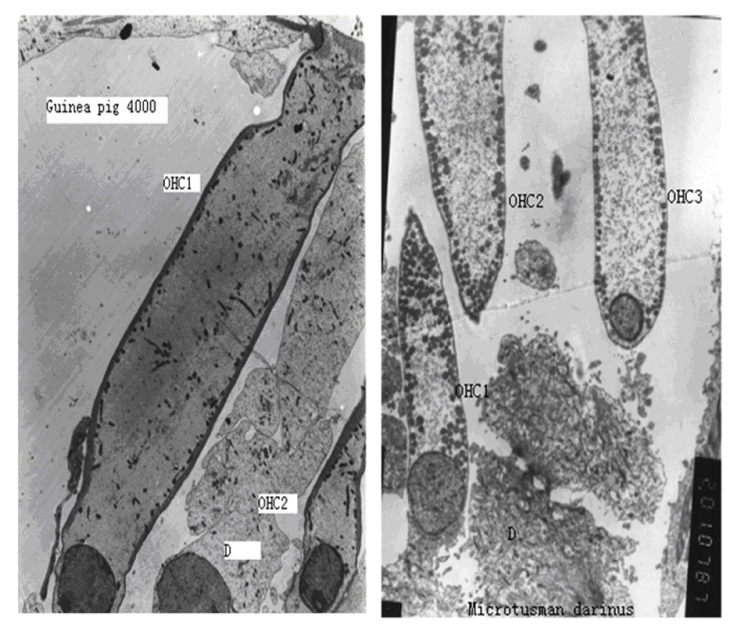

Figure 8. Transmission electron micrographs of the cochlear $\mathrm{OHC}$ structure of guinea pigs and Microtus mandarinus. Guinea pig cochlear $\mathrm{OHC1}$ possessed a slender and cylindrical shape in the hook region, whereas $M$. mandarinus $\mathrm{OHC} 2$ and $\mathrm{OHC} 3$ were cylindrical and the location of their nucleus was in its normal position near the base pole. Magnification: 4000X.

The OHC bodies of the Hipposideros and Rhinolophidae bats were cambiform. The cell body basal pole of the OHCs in the SI regions was not surrounded by the cup-shaped protrusion of the Deiters cells (Figure 9). The OHC bodies in the basal turn of Tadarida bat cochlea were not cylindrical, but were remarkably flask-shaped.

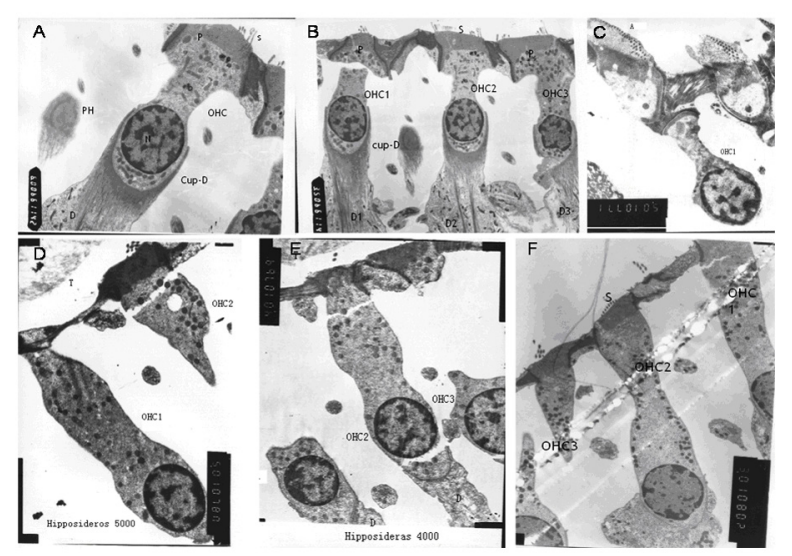

Figure 9. Transmission electron micrographs of the OHCs in the SI regions of the bat cochlea. Bat cochlear OHC body-shape in the SI and hook regions. A.-B. Hipposideros bat; C. Rhinolophidae bat; D.-E. The M. tadarida cochlear OHC1s were flask-shaped; F. Hipposideros bat cochlea OHC1 were cambiform-shaped. 


\section{$\mathrm{ABR}$ in bats}

The cochlear outputs and peripheral auditory sensitivity were assessed according to the ABRs. At $60 \mathrm{~dB}$ SPL, the hearing range of Hipposideridae extends from 3-48 kHz, with the best sensitivity at $32 \mathrm{kHz}$. The hearing range of Rhinolophus was from $6-32 \mathrm{kHz}$, with the best sensitivity at $24 \mathrm{kHz}$, and an ABR threshold of $10 \mathrm{~dB}$ SPL. The hearing range of $M$. schreibersii parvipes extends from 12-48 kHz, with the best sensitivity at $24 \mathrm{kHz}$ and an ABR threshold of $25 \mathrm{~dB}$ SPL. Compared with other mammals, the hearing range of the ICR mice extends from 4-32 kHz, with the best sensitivity at $16 \mathrm{kHz}$ and an ABR threshold of $15 \mathrm{~dB}$ SPL. The hearing range of $M$. mandarinus extends from $2-12 \mathrm{kHz}$, with the best sensitivity at $8 \mathrm{kHz}$ and an ABR threshold of $20 \mathrm{~dB}$ SPL. A comparison of the ABR thresholds is shown in Figure $10 \mathrm{a}$ and $\mathrm{b}$.

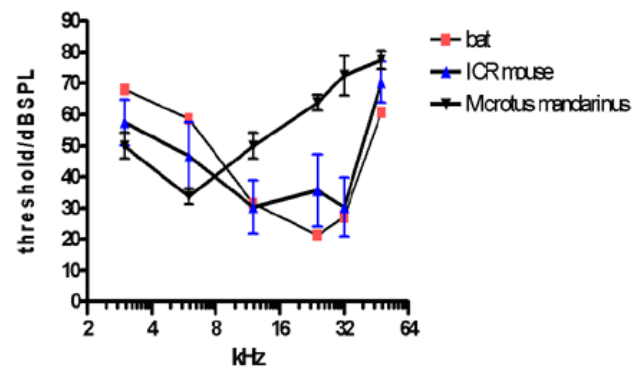

A

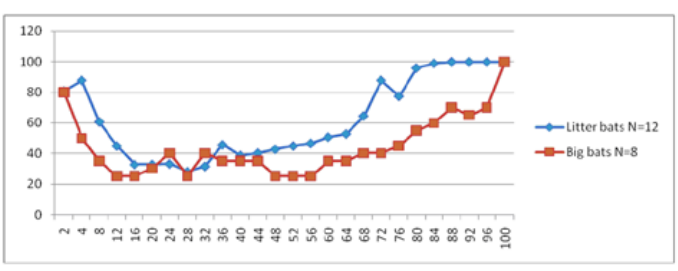

B

Figure 10. $A B R$ thresholds and $A B R$ audiograms. A. ABR threshold comparison: bats $(N=6)$, ICR mouse $(N=$ $14)$, and Microtus mandarinus $(\mathrm{N}=4)$; B. Hipposideros pratti Thomas bat ABR audiograms.

\section{DISCUSSION}

Bat survival depends on the acuity of their echolocation capabilities. Their auditory system has evolved special anatomic and physiologic features. Mammalian OHCs are slender cylindrical structures with fairly uniform diameters $(8-10 \mu \mathrm{m})$, and with lengths ranging from $20-30 \mu \mathrm{m}$ in the high-frequency cochlear base to approximately about $80-100 \mu \mathrm{m}$ in the lowfrequency apex (Dallos et al., 1991). Zajic and Schacht (1987) isolated OHCs from guinea pigs, chinchillas, rats, mice, and gerbils. They found that the morphological features of these OHCs were similar and that the cells were cylindrical. The apical length of the OHCs varied from $60-80 \mu \mathrm{m}$ in guinea pigs, whereas they were $20-40 \mu \mathrm{m}$ in chinchillas. The basal length varied from $7 \mu \mathrm{m}$ in mice and up to $10 \mu \mathrm{m}$ in other species. The OHCs of the mustached bat ranged from $12-13 \mu \mathrm{m}$ throughout the basal $80 \%$ of the cochlear length to a maximum of $20 \mu \mathrm{m}$ at the apex. The size of the OHC stereocilia ranged from 0.7-0.8 $\mu \mathrm{m}$ throughout the basal, which was $60 \%$ of the cochlear length, and reached a maximum of $2.2 \mu \mathrm{m}$ at the apex (Kachar et al., 1986). Vater et al. (1992) found that the receptor cells, IHCs and OHCs, of the echolocating horseshoe bat $R$. rouxi possessed typical mammalian shapes, but the OHCs were extremely short (length: $12-15 \mu \mathrm{m}$ in the basal turn and up to $28-30 \mu \mathrm{m}$ in the apical turn). Vater and Siefer (1995) observed that the OHCs throughout the cochlea of T. brasiliensis were cylindrical. In the present study, the OHC bodies were either round or flask-shaped. This 
novel finding highlights a new specialized feature of the Hipposideridae and Rhinolophus bats. Our other findings also revealed the short stereocilium bundles in the OHCs, the exaggerated length of the Deiters cell phalanges, and the specialized shape of the cup formation of the Deiters cell bodies that housed the bottom of the OHC (Figure 4). These results agree with those of Vater and Kǒssl. The shape of the apical OHCs conformed to the typical mammalian scheme. However, the basal OHCs were much shorter and ball-shaped. Their shape may have resulted from increased cell turgor because of the hypoosmotic extracellular solution (Reuter et al., 1994). In contrast, the basal OHCs from the cochlea of the horseshoe bat were cylindrical when examined under electron microscopy (Vater et al., 1992).

Many bat species produce isolation calls with peak energies between 10 and $25 \mathrm{kHz}$, corresponding to the frequency of the region with the highest sensitivity in these species (Macías et al., 2006). The ABR data suggested that there is a first sensitivity frequency region at $10-24 \mathrm{kHz}$ of the cochlea. The bat cochlea is specialized for very fine frequency resolution in the $\mathrm{CF}_{2}$ range. The specialized, SI region of the mustached bat cochlea is part of one of the most highly tuned and resonant biological structures. The SI and adjacent $\mathrm{CF}_{2}$ regions, which together constitute the cochlear fovea of the mustached bat, are specialized for analyzing the Doppler-shifted echoes of the first harmonic $(61 \mathrm{kHz})$ of the $\mathrm{CF}_{2}$ component of their echolocation call, which is used to measure target velocity and to detect the wing beats of insects (Russell et al., 2003).

In the SI region, the cochlear micromechanics may function in 2 different modes, including a conventional traveling wave that leads to shear displacement between the BM and $\mathrm{TM}$ and neuronal excitation for $62-70 \mathrm{kHz}$. In addition, the SI region responds to $61-62 \mathrm{kHz}$, with a resonance based on standing waves. Thus, this region preprocesses signals that are represented more apically in the $\mathrm{CF}_{2}$ region of the cochlea (Russell et al., 2003). The SI region, where signals between 61 and $72 \mathrm{kHz}$ are processed, is morphologically specialized and has a thickened BM and club-shaped TM, which interact to produce sharply tuned resonance (Russell et al., 2003). This resonance is detected by the hair cells in the $\mathrm{CF}_{2}$ region (Vater et al., 1992). Shifts in the frequency and delay tuning are all centrifugal only in the highly specialized subsystems of mustached bat for processing Doppler shifts or echo delays (Suga, 2008). In this context, the cochlear amplifier of some echolocating mammals remains functional for frequencies well above $100 \mathrm{kHz}$. Given that the receptor potential of hair cells in vivo rapidly decreases with frequency, there is some controversy as to whether the cochlear amplifier can function at the highest frequencies, as well as its underlying mechanism. In addition, with the OHCs embedded in the supporting structures in vivo, force generation at high frequencies may not induce cellular motility but instead cause stiffness changes, which enhance tuning (Kössl, 1997). We hypothesize that variation in the OHC body shapes in the cochlear fovea may be related to the high frequency function of the auditory response. The corticofugal system likely modulates the activity of cochlear hair cells through inhibitory olivocochlear neurons (Suga et al., 2000). The mustached bat emits a CF sound at $61 \mathrm{kHz}$ to acquire velocity information, as shown by its behavioral responses to Doppler-shifted echoes. To investigate whether these characteristics are based on cochlear properties, Macías et al. (2006) recorded the distortion product otoacoustic emissions and calculated their threshold curves. Cochlear sensitivity was observed between 20 and $50 \mathrm{kHz}$ and between 15 and $60 \mathrm{kHz}$ in both species. The behavioral threshold maxima separate the audiograms into 2 domains, indicating the separate processing of low-frequency social calls and high-frequency echolocation signals. The shape of these audiograms and the emergence of a threshold maximum are not due to cochlear properties, as 
shown in the present study. Xie and Henson (1998) concluded that tonic medial olivocochlear activity is induced by natural vocalizations and roost (where the bat sleeps) noises, and this activity likely regulates and protects the highly resonant cochlear partition reflex medial olivocochlear activity, causing a substantial reduction in the decay time of the CM produced by ringing. This may be the result of variations in the length and/or stiffness of the $\mathrm{OHC}$, which are generally thought to play a role in regulating the mechanical properties of the vibrating partition of the cochlea (Brownell et al., 1985). Previous studies demonstrated that mammalian $\mathrm{OHCs}$ are cylindrical or test tube-shaped and that they adapt to variations in length. Cylindrical mammalian OHCs of the inner ear produce rapid, force-generating length changes in response to electrical stimulation (Brownell et al., 1985). The sound-induced motility of isolated cochlear OHCs is frequency-specific (Dulon et al., 1989). However, the OHC bodies were found to be round, ball-shaped, or flask-shaped in the present study. These results are related to the bat cochlear active mechanics. We hypothesize that the OHC motion consists of 2 positions. One is located on the apex of the OHC (the neck of a bottle), which varies in length, causing contact between the BM and the TM. The second is at the base of the OHCs (the basal of bottle), indicating that the variation in the motion of contractions and the Deiters cell phalanges causes separation of the BM from the TM in the forward apex position of the cochlea. The highly specialized non-cylindrical morphology of the OHCs is complete and fit for active OHC motion tuning and echolocation in the SI region of the bats (Figure 1). We first found that the OHCs of bats were flask-shaped and cambiform in the SI to CF2 region of the cochlea. This specialized OHC shape has important significance in responding to highfrequency signals.

The morphology of the cochlear OHCs may be specifically designed to enable their contraction/elongation. In the organ of Corti, each $\mathrm{OHC}$ is mechanically coupled to the reticular lamina at the apex of the cell and to the Deiters cells at the base, whereas the cylindrical cell body lacks any contact with the adjacent cells. This suggests that structural alterations in the cytoplasmic membrane and the submembrane cortex occur under photo-irradiation, and that these structures are important for maintaining the elongated cylindrical shape of the OHCs, possibly by maintaining intracellular hyperosmolarity (Dulon et al., 1989). Mammalian OHCs are slender cylindrical structures with fairly uniform diameters and are very long, extending from the cochlear base to the apex. However, the reason the bat cochlear OHCs in the SI region are flask and cambiform shaped has not been elucidated thus far. One reason may be that very few studies have examined bat cochlear hair cells (Vater et al., 1992; Vater and Siefer, 1995). Moreover, only the cochlear apex turn OHCs have been observed because of the difficulty of isolating the OHCs from the cochlear basal turn (Housley and Ashmore, 1992). We hypothesize that all mammalian cochleae exhibit variations in $\mathrm{OHC}$ shape from the cochlear base turn to the apex turn. This is of particular interest in studying the variations in the highand low-frequency regions in the OHCs. OHCs presumably exhibit variations in motility and in cylindrical length in response to low-frequency auditory stimulation, possible alterations in its length, and they may oscillate in response to the high-frequency auditory stimuli. However, this hypothesis requires further investigation. In bats, there are multiple structural adaptations in both the OHCs and supporting cells, which may be crucial for effective amplification of high-frequency stimuli. In $R$. rouxi, the very short $\mathrm{OHC}$ stereovilli rows showed exaggerated angles of inclination against each other, which may lead to increased stiffness (Vater et al., 1992). The rigid reticular lamina and the formation of Deiters cups found in Carollia, which is described in detail for Rhinolophus, may considerably increase the stiffness of the whole organ 
of Corti and thus facilitate mechanical interactions at high frequencies (Reuter et al., 1994). In this study, we demonstrated that bat OHCs were flask-shaped and cambiform or ball-shaped in the $\mathrm{SI}$ to $\mathrm{CF}_{2}$ regions of the cochlea. However, the fast $\mathrm{OHC}$ movements were presumably quite small at ultrasonic frequencies and should be investigated further to determine how they participate in amplifying and sharpening cochlear responses in vivo. These data suggest that $\mathrm{OHC}$ body shapes with specialized features are adaptations for ultra-high-frequency echolocation in bat cochleae.

\section{Conflicts of interest}

The authors declare no conflict of interest.

\section{ACKNOWLEDGMENTS}

Research supported by the National Natural Science Foundation of China (Grant \#39970785) and the International Collaborate Communication Program (Grant\#30210103151). We thank Zhang from the Institute of Zoology, Chinese Academy of Sciences of Beijing for his help in the identification of the bat species. The illustration about afferent nerve fibers and the spiral ligament was kindly provided by M. Vater.

\section{REFERENCES}

Bohn KM, Boughman JW, Wilkinson GS and Moss CF (2006). Auditory sensitivity and frequency selectivity in greater spear-nosed bats suggest specializations for acoustic communication. J. Comp. Physiol. A Neuroethol. Sens. Neural Behav. Physiol. 190: 185-192.

Brownell WE, Bader CR, Bertrand D and de Ribaupierre Y (1985). Evoked mechanical responses of isolated cochlear outer hair cells. Science 227: 194-196.

Bruns V and Schmieszek E (1980). Cochlear innervation in the greater horseshoe bat: demonstration of an acoustic fovea. Hear. Res. 3: 27-43

Dallos P, Evans BN and Hallworth R (1991). Nature of the motor element in electrokinetic shape changes of cochlear outer hair cells. Nature 350: 155-157.

Dulon D, Zajic G and Schacht J (1989). Photo-induced irreversible shortening and swelling of isolated cochlear outer hair cells. Int. J. Radiat. Biol. 55: 1007-1014.

Housley GD and Ashmore J (1992). Tonic currents of outer hair cells isolated from the guinea-pig cochlea. J. Physiol. 448: 73-98.

Kachar B, Brownell WE, Altschuler R and Fex J (1986). Electrokinetic shape changes of cochlear outer hair cells. Nature 322: $365-368$.

Kirkegaard M and Jørgensen JM (2000). Continuous hair cell turnover in the inner ear vestibular organs of a mammal, the Daubenton's bat (Myotis daubentonii). Naturwissenschaften 87: 83-86.

Kössl M (1997). Sound emission from cochlear filters and foveae- does the auditory sense organ make sense? Naturwissenschaften 84: 9-16.

Li G, Wang JH, Rossiter SJ, Jones G, et al. (2008). The hearing gene prestin reunites echolocating bats. Proc. Natl. Acad. Sci. U. S. A. 105: 13959-13964.

Macías S, Mora EC, Coro F and Kössl M (2006). Threshold minima and maxima in the behavioral audiograms of the bats Artibeus jamaicensis and Eptesicus fuscus are not produced by cochlear mechanics. Hear. Res. 212: 245-250.

Reuter G, Kössl M, Hemmert W, Preyer S, et al. (1994). Electromotility of outer hair cells from the cochlea of the echolocating bat, Carollia perspicillata. J. Comp. Physiol. A 175: 449-455.

Russell IJ and Köss1 M (1999). Micromechanical responses to tones in the auditory fovea of the greater mustached bat's cochlea. J. Neurophysiol. 82: 676-686.

Russell IJ, Drexl M, Foeller E, Vater M, et al. (2003). The development of a single frequency place in the mammalian cochlea: the cochlear resonance in the mustached bat Pteronotus parnellii. J. Neurosci. 23: 10971-10981.

Genetics and Molecular Research 14 (3): 9530-9542 (2015)

CFUNPEC-RP www.funpecrp.com.br 
Suga N (2008). Role of corticofugal feedback in hearing. J. Comp. Physiol. A Neuroethol. Sens. Neural Behav. Physiol. 194: 169-183.

Suga N, Gao E, Zheng Y, Ma X, et al. (2000). The corticofugal system for hearing: recent progress. Proc. Natl. Acad. Sci. U. S. A. 97: 11807-11814.

Vater M and Siefer W (1995). The cochlea of tadarida brasiliensis: specialized functional organization in ageneralized bat. Hear. Res. 91: 178-195.

Vater M, Lenoir M and Pujol R (1992). Ultrastructure of the horseshoe bat's organ of Corti. II. Transmission electron microscopy. J. Comp. Neurol. 318: 380-391.

Xie DH and Henson OW Jr (1998). Tonic efferent-induced cochlear damping in roosting and echolocating mustached bats. Hear. Res. 124: 60-68.

Zajic G and Schacht J (1987). Comparison of isolated outer hair cells from five mammalian species. Hear. Res. 26: 249256. 